\title{
EL TEMA DE LA MORFOLOGÍA URBANA EN LA HISTORIA DEL PENSAMIENTO GEOGRÁFICO
}

\author{
Vicente Bielza de Ory \\ Dpto. de Geografía y Territorio, Universidad de Zaragoza \\ vbielza@unizar.es
}

\begin{abstract}
Resumen: La morfología urbana que a principios del siglo XX empezó siendo un tema central de la geografía urbana, cuando se enfocaba desde una concepción paisajística, ligada al cuadro natural y a la evolución histórica, sufrió un estancamiento con la New Geography, que desde una óptica espacial, cuantitativa y economicista se centró más en las cuestiones estructurales. La recuperación del paisaje en los años ochenta y los trabajos de los geógrafos en equipos multidisciplinares de planificación han mejorado la situación de la morfología, especialmente en relación con los cascos históricos y paisajes postindustriales.
\end{abstract}

Palabras clave: Morfología urbana, historia del pensamiento geográfico.

\begin{abstract}
The Urban morphology, which in the early twentieth century began as a central theme of urban geography, when it was approached from a conception based on the landscape, linked to the natural framework and historical evolution, was stagnated with the New Geography, which had a spatial perspective, economistic, quantitative and more focused on structural issues. The recovery of the landscape in the eighties and the work of geographers in planning multidisciplinary teams have improved the situation of the morphology, especially in relation to the historic urban areas and post-industrial landscapes.
\end{abstract}

Keywords: urban morphology, history of geographical thought.

Recibido: 2-11-10. Aceptado: 2-2-12. 
A lo largo del siglo XX este tema apenas ha superado el estadio descriptivista, como señalaba Carter en los años setenta (Carter, 1972) y Estébanez a finales de dicha centuria (Estébanez ,1993) Cuando a principios del pasado siglo la Geografía Urbana empezaba a despuntar como materia científica, tanto en la más determinista geografía alemana como en la opción posibilista francesa, la forma urbana se enfocaba desde una concepción paisajística, ligada al cuadro natural y a la evolución histórica. En general, dicha visión centraba el objetivo de la geografía en la forma y la distribución de los fenómenos sobre la superficie terrestre, lo que significaba, en particular, que la geografía urbana debía poner la forma urbana en el primer lugar de sus investigaciones. Ello no fue así, como veremos, a lo largo del pasado siglo, especialmente en los años sesenta y setenta, aunque la recuperación del paisaje en los ochenta y los trabajos en equipos multidisciplinares de los geógrafos hayan mejorado la situación. A principios del siglo XXI el descubrimiento del paisaje como objeto de estudio e instrumento de ordenación territorial por otros expertos y por políticos preocupados por el desarrollo sostenible, ha llevado a partir de la Convención Europea del Paisaje (Florencia, 2000) a identificar y calificar los "paisajes naturales, rurales, urbanos y periurbanos" con objeto de protegerlos, gestionarlos y ordenarlos para mejorar la calidad de vida (Zoido, 2002). Como en otros viejos conceptos de la geografía, han tenido que ser otros especialistas los que nos ayuden a recuperar visiones holísticas, propias de nuestra materia y esencialmente fecundas. Esperemos que el gran interés por el paisaje, tan defendido ahora por otros urbanistas y ordenadores del territorio, sirva para avanzar en la concepción científica de la morfología del paisaje cultural por excelencia y el que más afecta a la calidad de vida del ciudadano, cual es el paisaje urbano, que adopta su mejor expresión en los centros históricos, sobre los que los geógrafos muestran una renovada atención que reparten con los paisajes postindustriales, en los que la intervención de geógrafos planificadores, como P. Hall, ha servido para enriquecer el pensamiento geográfico y las formas postindustriales de planificar. Para ello conviene conocer los inicios de la morfología urbana y su evolución en la geografía.

\section{El enfoque clásico}

La escuela alemana, iniciada por Ratzel, quien ya se ocupó en su Antropogeografía (Ratzel, 1882) de la ciudad, para estudiar la morfología de los paisajes en general y de los urbanos en particular se apoyó en el concepto de kulturlandschaft, expresado por Otto Schlüter en 1899. La línea paisajística del landschaft fue bien desarrollada por Siegfried Passarge a principios de siglo y concretada en el plano urbano por E. Oberhumm en 1907 y en la tipología edificatoria de Viena por H. Hassinger a partir de 1912 (Hassinger, 1912). 
Las aportaciones de los primeros investigadores alemanes serían recogidas en el manual de Norbert Krebs sobre geografía humana de 1920 (Krebs, 1931), en cuyo capítulo X trataba de las "Formas y evolución de los medios urbanos", y donde se refleja el menor nivel de los estudios urbanos respecto de otras partes de la geografía. Se inicia el capítulo urbano describiendo, de forma elemental, para después diferenciar tipológicamente, las características urbanas: En el núcleo urbano la plaza del mercado, la iglesia y la casa consistorial ${ }^{1}$ ocupan una posición preferente, accesible, fácilmente por todas partes. Las casas se aglomeran y son más altas ${ }^{2}$. A continuación Krebs se ocupa del trazado de las calles, de los barrios y su carácter propio, de la huella de las antiguas murallas y en general del pasado histórico. Trata después del emplazamiento topográfico y de la influencia en el plano y forma de la ciudad. A partir de aquí apunta el geógrafo alemán la uniformización que se observa en las nuevas edificaciones de Berlín y Viena, que no son felizmente, el tipo predominante. La diversidad de la herencia preindustrial es lo que permite diferenciar las ciudades con sus respectivas formas ${ }^{3}$ : europeo-occidentales, europeo-orientales, mediterráneas, orientales y americanas. Hay pues, en esta, como en otras geografías urbanas alemanas de la época, una tipología muy descriptivista y cualitativa de las formas urbanas, propia de los comienzos científicos de una disciplina.

Hassinger, Passarge, Bobek y la escuela de Viena siguieron haciendo, en la misma línea, aportaciones valiosas a la morfología urbana hasta después de los años cincuenta, estudiando sus propias ciudades y las de otros paises ${ }^{4}$. A partir del paisaje, se explicaba la morfogénesis del plano, su estructura interna y se establecían tipologías 5 , apoyándose en atlas urbanos.

En la escuela francesa el iniciador de los estudios urbanos fue R. Blanchard, quien, desde las ideas vidalianas, en 1912 abordó el estudio urbano de Grenoble ${ }^{6}$ partiendo de los conceptos de situación y emplazamiento (capítulos I a III) que condicionaron

\footnotetext{
${ }^{1}$ P. George en Précis de geographie urbaine (1960) define las ciudades europeas como una "familia" urbana caracterizada, también, por esos tres elementos: iglesia, ayuntamiento y mercado.

${ }^{2}$ Luego hace una relación de la procedencia de los materiales de construcción, que ante los incendios y el desarrollo de las comunicaciones superan el abastecimiento del entorno, frente a la vivienda rural, utilizando piedra o ladrillo alógenos

3 "Las europeoccidentales descritas anteriormente, de las orientales de casas pequeñas y dispersas (de Rusia, Polonia o Hungría) y, a su vez, de las mediterráneas, de edificación densa y hasta laberínticas, o de las ciudades orientales, situadas en oasis o de las americanas, tiradas a cordel"

${ }^{4}$ Resultó muy valioso para España el estudio de O. Jessen sobre los paisajes urbanos españoles en 1947

${ }^{5}$ W. Geisler ya en la década de los veinte clasificó las ciudades alemanas por su emplazamiento, planos $\mathrm{y}$ edificios

${ }^{6}$ El párrafo final resume esta dialéctica:"Así de un extremo a otro de la existencia de la ciudad, nosotros encontramos el efecto de los mismos fenómenos. Desde su nacimiento hasta su expansión actual, Grenoble es la ciudad de la confluencia de los ríos y de sus valles. A despecho de las vicisitudes humanas. La naturaleza toma siempre sus derechos, incluso sobre el organismo complejo que es una ciudad".
} 
la evolución urbana, estudiada desde sus orígenes (cap. IV) y hasta el siglo XX (cap. V). La forma es el resultado del condicionamiento natural del emplazamiento primitivo y de la evolución histórica? ${ }^{7}$.

A partir de la tesis de Blanchard, las escuelas dependientes de la francesa, afrontaron las investigaciones urbanas para llegar a la morfología, siguiendo un esquema similar al suyo: situación, emplazamiento, génesis histórico-urbana, funciones, morfología (Blanchard, 1912) ${ }^{8}$. La morfología se apoyaba a su vez en el plano, las edificaciones, la función de las mismas y el uso del suelo. La uniformidad de los materiales de construcción, ya advertida por Krebs y consolidada en la ciudad industrial del XX, fue uno de los motivos para que pasara a un segundo plano esta consideración del paisaje urbano, trasladada del rural.

Berdoulay y Soubeyran han demostrado recientemente (Berdoulay, V. et Soubeyran, O., 2002 ) cómo el urbanismo francés abandona la variable ecológica antes de la II Guerra Mundial, después de que en sus inicios, de la mano de los geógrafos vidalianos, influyera de tal manera que el arquitecto Jaussely, primer presidente de la Sociedad de Urbanistas Franceses, SFU (1924) dijera que los geógrafos son los verdaderos padres del urbanismo. En la revista la Vie urbaine y en el Insto ${ }^{\circ}$ de urbanismo de la Sorbona los geógrafos fueron relegados por arquitectos, juristas e historiadores a explicar el medio físico antes de fundarse la ciudad, cuya evolución posterior quedaba en manos de los historiadores. Quizá por ello, la evolución morfológica de la ciudad no fue en adelante un objetivo prioritario para los geógrafos franceses y tampoco para los latinos en general.

La geografía urbana clásica, junto al enfoque morfológico, apoyado en los condicionantes ecológicos (situación-emplazamiento) y taxonomizado desde la geometría de los planos, tenía en cuenta la evolución histórica, a modo de explicación genética de la forma. Hay que destacar la obra de P. Lavedan, tanto en su versión directamente histórica, como su geografía de las ciudades (Lavedán, 1936), que tanto influyó en los geógrafos franceses y españoles, de modo parecido a como la de John Reps impactó en el mundo anglosajón (Reps, 1965).

Cuando se quiere pasar de las monografías a los estudios de conjunto de la geografía urbana se buscan tipologías de formas urbanas, más allá de la base ecológico-

\footnotetext{
${ }^{7}$ Blanchard, 1912, p. 159. Ver la magnífica semblanza que hace V. Berdoulay de esta cuestión en el cap. VII "Dans la ville comme un tout: la stratégie narrative de R. Blanchard a propos de Grenoble" in Aux débuts de l'urbanisme français, 2001, p. 83.

${ }^{8}$ Así se llevaron a cabo las primeras tesis doctorales en España a finales de los cincuenta por parte de Joaquín Bosque (Granada) y de Ana Ma Navarro (Zaragoza) bajo la dirección de José Manuel Casas Torres y a mitad de los sesenta las primeras tesinas sobre pequeñas ciudades, como la dirigida por el primer discípulo de aquel, Alfredo Floristán ( V. Bielza: Estella, estudio geográfico de una pequeña ciudad navarra, 1968).
} 
cultural, apoyándose casi exclusivamente en el plano, de modo que la forma tridimensional tiende a reducirse a la bidimensional que se desprende del plano. La gran influencia de P. Lavedan en la Geografía urbana francesa de los años 30 y sucesivos hace que los manuales de Chabot, Beaujeu-Garnier y otros reproduzcan las taxonomías de Lavedan. Lo que harán también las escuelas dependientes de la francesa. Esta servidumbre, repitiendo las grandes líneas y también algunos errores de Lavedan' ${ }^{9}$, y la complejidad del tema arrastraron una perpetuación de lo expuesto por el maestro galo, incluso cuando se pase a otros planteamientos conceptuales y metodológicos. Entre tanto, en otras partes de la geografía, como la geomorfología o la geografía agraria, se pudo avanzar del descriptivismo, a la taxonomía y luego a la génesis y a los modelos de las formas de relieve o de los paisajes agrarios. Veamos a continuación cómo se repiten en los manuales de geografía urbana las meras clasificaciones de planos para justificar la morfología urbana, prescindiendo de las formas tridimensionales urbanas y de otros aspectos relativos a la morfogénesis o de la influencia cultural.

G. Chabot en 1948 en su libro Les villes, que parte del estudio de la función, identifica la forma de la ciudad con el marco de la cité, donde a partir del emplazamiento estudia el plano, condicionado por la función y por el emplazamiento. Los planos sistemáticos o preconcebidos, frente a los espontáneos e irregulares, según la antinomia de Lavedan, los dividió, a su vez, en aquellos que reflejan influencias técnicas y religiosas (pueblos circulares de los eslavos), influencias militares (castrum romano) o los que modernamente se concibieron buscando una racionalidad, como ya hiciera Vitruvio en busca de la ciudad ideal. Plano ortogonal, plano radioconcéntrico y plano en estrella se reparten ventajas e inconvenientes, a juicio de Chabot.

Tricart, buen geomorfólogo físico, en 1954 al abordar el habitat urbano (Tricart, 1954) llega a una clasificación más compleja de la forma urbana pero también exclusivamente apoyada en la geometría del plano, diferenciando ciudades homogéneas de las heterogéneas y entre las primeras las planificadas de las que no lo son ${ }^{10}$.

Derruau en 1961, dentro de su geografía humana (Derruau, 1961) afronta la morfología urbana en el epígrafe de "los barrios de las ciudades", después de tratar de la situación, el emplazamiento y el crecimiento. Inicialmente afirma que el aspecto formal de las ciudades varía de un barrio a otro: las casas difieren unas de otras, cada barrio tiene sus características propias, en resumen el plano de conjunto varía en cada una de las aglomeraciones. Esta inicial afirmación que parece conducir a la

\footnotetext{
${ }^{9}$ P. ej. en relación con el origen de la bastida y la cita de Lacarra que exponemos en Bielza de Ory, V.: "La ciudad ortogonal aragonesa del Camino de Santiago y su influencia en el urbanismo regular posterior", 2000 .

${ }^{10}$ A) Homogéneas. 1-Planificadas: 1.1. rectangular: -lineal-diagonal-paralelo-damero. 1.2. radioconcéntrica: -estrella -circular .2-No planificadas: -Fortaleza -Estrella -Irregular. B) Heterogéneas o complejas: 1Replanificadas. 2-Polinucleares. 3-Con un patrón-red. 4-Esféricas: -concéntricas -radiales.
} 
imposiblidad de un enfoque nomotético, es matizada con los intentos taxonómicos en relación con los edificios urbanos ${ }^{11} \mathrm{o}$ los barrios ${ }^{12}$. Pero será en el apartado correspondiente al plano urbano, donde quiera resolver la cuestión de la forma urbana en su conjunto. Tras abordar la influencia del emplazamiento trata de los planos racionales con sus ventajas e inconvenientes, dividiéndolos en formas de: tablero de ajedrez, de estrella y radioconcéntricos, es decir que acaba apoyándose en los tipos de plano para tipificar las formas urbanas.

Beaujeu-Garnier y Chabot en 1963, en el que se puede considerar como el manual maduro de la geografía urbana francesa en su etapa clásica (Beaujeu-Garnier et Chabot, 1963) resuelven la morfología urbana, como lo hiciera anteriormente el segundo, después de las funciones, en un capítulo titulado "el plano y la extensión de las ciudades”, donde divide el mismo en: ortogonal, radio-concéntrico y lineal.

También los geógrafos anglosajones de la primera mitad del siglo XX identificaron bastante la morfología urbana con las tipologías de planos, aunque de manera algo distinta y sin separarse del medio natural y de la práctica urbanística, en la que algunos, como Taylor, colaboraron con ingenieros y arquitectos. En Inglaterra la temprana urbanización y metropolización condujo a los geógrafos, como a otros científicos, a interesarse por lo urbano y lo suburbano desde finales del siglo XIX ${ }^{13}$. No obstante los geógrafos británicos -como ha recordado Capel- limitaron el estudio de la forma urbana a la evolución del plano y la clasificación de los distintos tipos de estos (Capel, 2002). La línea de la geografía cultural indujo al estudio de la tipología de ciudades en Europa, que consiguió sus mejores logros en la obra de R.E.Dickinson sobre la morfología de la ciudad occidental, en la que intenta definir los rasgos comunes de dicha ciudad y su morfología, clasificando los sistemas básicos de plano en tres grupos: irregulares, radio-concéntricos y rectangulares o en damero (Dickinson, 1951)

El libro de G. Taylor, escrito en 1946, después de casi cuarenta años de experiencias en trabajos urbanísticos, iniciados en Canberra, es uno de los primeros manuales anglosajones de geografía urbana (Taylor, 1946). Desde los casos particulares por él conocidos, sobre todo de las ciudades nuevas anglosajonas, llega a ciertas generalizaciones. En primer lugar entiende como clave de la ciudad la influencia dominante del medio, por ello la primera clasificación es por el emplazamiento, aunque también cuenta con la evolución histórica ${ }^{14}$. Por tanto Taylor arranca, como

\footnotetext{
${ }^{11}$ Locales y viviendas y estas clasificadas en modestas, bloques de pisos, individuales para pudientes...

${ }^{12}$ Clasificados funcional o formalmente, en este caso por el aspecto de monumentos, viviendas, disposición viaria y espacios verdes.

${ }^{13}$ Recordemos que el primer plan de ordenación urbanística y del territorio fue el del Gran Londres en 1909 .

${ }^{14}$ Según Taylor las ciudades industriales atraviesan tres fases de desarrollo; infantiles, juveniles y modernas, pero aún en la misma fase de madurez se siguen diferenciando por el distinto emplazamiento (Nueva York y Chicago, p. ej.).
} 
Blanchard, pero de modo más determinista, del medio topográfico, y de la evolución histórica. Las formas se deben a determinantes topográficos ${ }^{15}$, aunque reconoce otros factores y funciones ${ }^{16}$. También se ocupa de los planos, clasificándolos dialécticamente en damero y "distintos del tablero de damas", ya que por haber iniciado sus estudios en las ciudades nuevas anglosajonas y no en las europeas, como los geógrafos anteriores, lo normal, para él, es que "el plano de muchas poblaciones consista meramente en una extensión del sistema en tablero de damas, empleado en la división del campo (townships)"17. Sin embargo el plano en damero conlleva problemas. Por ello considera Taylor que no es recomendable para planificar las nuevas ciudades del siglo XX, cuestión por la que ya en 1910 para Canberra, colaboró en la concepción de un plano en tela de araña, polinuclear, radio-concéntrico y adaptado al medio natural (entre colinas).

Dickinson, a pesar de las aportaciones propias y las de otros geógrafos como Taylor (quien, a su vez, tuvo en cuenta las suyas) opinaba a finales de los cuarenta que las contribuciones hechas desde la geografía anglosajona eran de baja calidad, debido a que "el enfoque ha sido más empírico que genético, y es este sólo el que permite el reconocimiento de lo importante" 18 .

\section{Los intentos morfológicos de las nuevas geografías de mitad del siglo XX}

Lo expresado por Dickinson sobre la baja calidad de las investigaciones era extensible a otras escuelas geográficas y durante dos décadas más, de tal modo que en 1962 el Symposium de la UGI sobre Geografía Urbana, celebrado en Lund, reconoció que siendo uno de los más antiguos y mejor conocidos aspectos de la geografía urbana, es al mismo tiempo, el único en que comparativamente se ha hecho menos progreso. Garrison culpó de ello a la falta de progreso en la teoría general y a la falta de buenos recursos de medición (Carter, 1972). La comparación con las innovaciones de otras partes de la urbana contempladas en Lund, como las nuevas aportaciones presentadas por Christaller a la teoría de lugares centrales, resultaba odiosa para la morfología. El problema quiso ser solventado por la New Geography, iniciada en la década de los cincuenta en los países nuevos anglosajones, herederos de ciudades muy homogéneas, predominantemente trazadas en damero, donde pronto se modelizaron aspectos tales como el sistema de ciudades o la estructura urbana, quedando estancada, en cambio, la forma urbana propiamente dicha en un estadio descriptivista-taxonómico.

\footnotetext{
${ }^{15}$ Factor geológico, ciudades en llanura, fluviales-portuarias, de montaña.

${ }^{16}$ Ciudades mineras, centros religiosos, poblaciones turísticas.

17 ... Tal sistema es útil en esta última aplicación por su simplicidad y por ser fácil la subdivisión de los bloques rectangulares, cuando una zona, se convierte en urbana...

${ }^{18}$ Tomado de Capel, op. cit., p. 27.
} 
De los tres elementos en que la geografía clásica desagregó el análisis de la forma urbana: plano, cuyo estudió priorizó, edificaciones y usos del suelo condicionados por las funciones (para muchos a considerar en otro apartado, el de estructura urbana), fue el tercero el más abordado por los seguidores de la Nueva Geografía (Bunge, 1962), pero apoyándose en unas teorías económicas del suelo, que exigen una superficie indiferenciada (isotrópica), como si no existiera un emplazamiento condicionante y un plano incidente en los usos del suelo y sin tener en cuenta la altura y los edificios. En el caso del plano el análisis cuantitativo se centró en el entramado viario, considerado como red topológica, susceptible de ser abordada mediante la teoría de grafos y en establecer tipos geométricos, así como características de tamaño, accesibilidad de los nodos, etc. Las características de los edificios como la altura, el número de pisos, la volumetría, el número y forma de los vanos se cuantificaron para establecer tipologías a partir del análisis factorial (Hagget, 1965)

Los análisis y teorizaciones parciales de usos del suelo, redes viarias y edificios no se consiguieron integrar, en modelos globales, para explicar la forma urbana y establecer predicciones. Por ello algunos buscaron las generalizaciones de la forma urbana a partir de los estudios previos de geógrafos provenientes de líneas inductivohistoricistas, practicadas en la escuela germánica, como Conzen, quien a partir del estudio histórico de Alnwick (Northumberland) dedujo un modelo que podía alcanzar un cierto grado de generalización, desde la consideración de las franjas periféricas de la ciudad (con elementos fijos y variables) y la diferenciación de fases de crecimiento y estancamiento.

Al llegar a la década de los 70 hay una insatisfacción generalizada, tanto en la geografía clásica francesa, que sigue reproduciendo la doble línea ecológica e historicista, como en la renovada geografía anglosajona. Así Johnson en su manual "Urban Geography" (Jonson, 1974) en que modeliza el sistema de ciudades y teoriza sobre la estructura urbana reconoce en cuanto a la forma urbana que a pesar del interés que este tema despierta entre los geógrafos desde hace mucho tiempo, se han escrito pocos trabajos que den cuenta de una manera completa del legado visible de las distintas ciudades, legado al que se refiere más arriba como morfología o paisaje urbanos. Ello se debe a la variedad de factores que concurren a la fijación de la morfología, que complican tanto la descripción como su explicación. Las tipologías más frecuentes son: la ciudad colonial, que suele dar lugar a plantas en damero; la ambición de monumentalidad para exaltar el poder mediante monumentos y planos en perspectivas o desde la concepción total radio-concéntrica de las nuevas capitales anglosajonas. La búsqueda de la ciudad ideal, desde las fortificaciones renacentistas a la ciudad jardín cierra las tipologías de planos.

Un mayor esfuerzo por superar el estadio descriptivo hizo Harold Carter, aún proviniendo de una formación clásica, en su "The Study of Urban Geography" (Carter, 
1974) al intentar incorporar a este aspecto de la geografía urbana las nuevas corrientes anglosajonas y pretender objetivos más generalizadores. Comienza afirmando que al término "paisaje urbano" en geografía urbana se le considera como expresión de toda la escena objetiva visible en el área urbana o la completa y subjetiva "imagen de las ciudades". Con ello incorporaba las, entonces recientes, investigaciones de Kevin Lynch sobre la imagen de la ciudad y la percepción de la misma (Lynch, 1960), Desde una u otra expresión la totalidad de la forma urbana puede ser considerada como un sistema formado por partes interrelacionadas, a juicio de Carter, que así introduce la concepción sistémica, que luego ve difícil de aplicar, a causa de la confusión que entraña el estudiar separadamente los tres elementos de la concepción clásica (plano, edificio y uso del suelo). Aceptando dichos fallos, considera separadamente dichos elementos, dedicando un capítulo al análisis del plano, en el que después de recordar las líneas tradicionales, preocupadas por los tipos de forma y por la evolución histórica, advierte una superación de la inducción desde la geografía cuantitativa con el modelo de Bunge (Bunge; 1962), relativo a la forma o la consideración del plano como red lineal, tal y como lo aborda Hagget (Hagget, 1965). La conversión del plano urbano en una red lineal y la aplicación de las correspondientes técnicas, pueden permitir la comparación cuantitativa de planos, pero no resolver la modelización de la forma de modo satisfactorio.

La tipología de planos aportada por los geógrafos clásicos seguía imponiéndose en aquellos que en los años 80 querían seguir hablando de morfología y no sólo de estructura urbana (usos y valor del suelo). De hecho la concepción de la ciudad-espacio en sustitución de la ciudad-lugar en los geógrafos europeos no fue tan radical como en los norteamericanos, de manera que aquellos, sobre todo los de tradición francesa, siguieron apoyando la morfología urbana en el plano y sus tipologías clásicas. Es el caso de Bastié y Dezert en L'espace urbain (Bastié et Dezert,1980) en que llegan al plano y la estructura urbana desde los conceptos de situación y emplazamiento y desde el suelo urbano y su valor. Hay un intento en dichos geógrafos galos de relacionar los tipos de plano con el contenido social y con los monumentos simbólicos para establecer cuatro tipos de planos en los centros preindustriales: radioconcéntrico, semi-radio-concéntrico (variante del anterior), ortogonal y plano en estrella. A ello se le añaden las tendencias recientes, es decir las del urbanismo industrial. La estructura urbana en relación al centro y la conexión entre forma y estructura completan un débil capítulo, poco satisfactorio, frente a otros, bien conseguidos, de dicho manual.

Los geógrafos cuantitativos de los países nuevos anglosajones, cuando en los años ochenta escriben sus manuales, van más lejos, al ningunear los tipos de planos y abordar directamente una geometría de la ciudad que en dichos países es de una gran sencillez: un CBD desarrollado en altura, rodeado de una zona de transición más baja y plurifuncional y de anillos residenciales de las clases medias y altas ubicadas en 
viviendas unifamiliares. El hecho de que el plano en damero haya sido, como ya demostrara Taylor, la regla de las ciudades nuevas anglosajonas parece permitir el obviar su estudio. Así Haggett plantea el modelo espacial interior de la ciudad norteamericana, de tan sencilla forma urbana, para abordar una geometría de los valores del suelo, que representados tridimensionalmente ofrecen también una figura central, virtual, de rascacielos (Haggett, 1983). Ello conduce inmediatamente a la estructura urbana de los usos del suelo para retomar lo morfológico, pero percibido desde la imagen mental. La comparación entre ciudades, una vez asentado el modelo más estructural que morfológico de lo que Haggett llama la ciudad de civilización occidental (que sólo es la de los países nuevos anglosajones) es afrontada desde los conceptos tomados del geógrafo físico Davis de estructura, proceso y etapa. La estructura inicial se apoya en la situación y el emplazamiento y luego se plantean procesos en el interior y con el exterior de la ciudad, que clasifican la población socialmente. Las etapas se refieren a las fases preindustrial ${ }^{19}$, industrial y postindustrial. La ciudad industrial es caracterizada por la estructura, especialmente de la densidad de población, que a partir del centro, donde al principio se registra el valor máximo, a través del tiempo tiende a comportarse como un cucurucho de helado que se va fundiendo al perder población su CBD, frente a lo que sucede con las del Tercer Mundo que incrementan su población en el centro. Para la ciudad postindustrial, Haggett se pregunta: “Qué tipos de formas sustituirán al actual centro urbano-industrial?". La respuesta es que se concentrarán los puestos de trabajo altamente representados en los sectores $3^{\circ}$ y $4^{\circ}$ con arreglo a las tendencias de las curvas. Es decir que la forma urbana para Haggett es la forma de las curvas que representan la densidad de población o del empleo especializado, olvidándose de la geometría real, del paisaje.

Tampoco los movimientos sociales y radicales que en los 70-80 invadieron algunas escuelas geográficas resolvieron satisfactoriamente la cuestión morfológica, a pesar de que abrieron nuevos campos en la geografía urbana, como hiciera David Ley (Ley, 1983) que permitían profundizar en asuntos tales como el del papel de los agentes sociales que, sin duda, impactan en la forma urbana, lo cual permite avanzar en el conocimiento de la morfogénesis pero no propiamente en una teoría de la forma. Teoría que intentarían formular los urbanistas europeos provenientes de la arquitectura, desde equipos multidisciplinares, normalmente invadidos por la sociología marxista, para dar respuestas a las demandas sociales de recuperación de los centros históricos, a partir de la crisis económica, constructiva y de modelo urbano que se abrió a mitad de los setenta.

\footnotetext{
${ }^{19}$ Según la obra de Haggett: Las ciudades preindustriales se caracterizaban por la muralla, el trazado viario regular, las edificaciones de una o dos plantas, salvo los edificios públicos (iglesias, mercado, palacio). Las funciones preindustriales, aunque mezcladas con los usos residenciales del suelo, daban lugar a agrupaciones por gremios en determinadas zonas o calles. Las clases sociales más acomodadas tendían a residir en el centro.
} 


\section{La renovación historicista y paisajística de la morfología}

La preocupación por los centros históricos suscitó en aquellos geógrafos que participaron en grupos pluridisciplinares de restauración, junto con los historiadores generales y del arte, la renovación de la línea historicista y, mas tarde, junto a los arquitectos, de la paisajística de la geografía, incorporando la atención a los agentes sociales, la percepción ante las decisiones de los mismos, la empatía y los valores simbólicos.

La renovación historicista de la geografía, después de los intentos teoréticos, supuso apoyarse en aquellos geógrafos que desde formaciones clásicas habían intentado generalizaciones o modelizaciones, como en el caso del citado Conzen, que fue punto de partida para las investigaciones de Whitehand, quien analizó la evolución de las formas construidas en relación con los procesos de innovación-difusión y las fases de expansión-recesión económica, ocupándose de la función desempeñada por propietarios y administración, tanto en las periferias urbanas como en el centro. Sus trabajos han suscitado un valioso grupo investigador sobre morfología urbana: Urban Morphology Group (Whitehand, 1987).

Otro geógrafo anglosajón que arranca de los historicistas anteriores es Carter, que a partir de un trabajo de Stanislawski, (1946) concluye una generalización: "las variables que forman el plano pueden ser independientemente aisladas de cualquier fase histórica”. Es posible, proponer una lista que incluya, por ejemplo, factores políticos, económicos, culturales y religiosos, pues aunque no actúan independientemente, la mayoría de los elementos del plano son producto de dos o más, o incluso de todos ellos. En el presente contexto pueden ser considerados separadamente. Carter en una época en que se habían primado los factores socioeconómicos pone el acento en los políticos y religiosos, subrayando el poco caso que se había hecho hasta entonces de los segundos ${ }^{20}$. Carter ejemplifica las influencias religiosas mostrando el contraste entre el trazado de Filadelfia, dentro de una Pensilvania concebida desde la cultura cuáquera, y el de las ciudades de Nueva Inglaterra que obedecen al puritanismo ${ }^{21}$. El contraste entre la colonización en el área pensilvana y la de los puritanos en Nueva Inglaterra puede relacionarse con las diferentes formas de culto. "Este aspecto de la influencia religiosa en el plano merece más atención de la que hasta ahora se le ha prestado. En áreas extra-europeas las fuerzas cosmológicas han sido particularmente

\footnotetext{
${ }^{20}$ Carter, 1972: Bajo el título de influencias políticas se incluyen aquellas fuerzas que se relacionan con la concentración del poder (persona o grupo).

${ }^{21}$ La población estaba libre de vasallaje (de una religión fija o de un lugar de culto) y pudo progresar rápidamente. Los amigos del libre culto encontraron en sus hogares los lugares agradables para reuniones, mientras sus contemporáneos trataban en Nueva Inglaterra sobre las normas para el emplazamiento adecuado de las capillas puritanas y de las viviendas colindantes.
} 
importantes y su significado dentro de Europa no ha sido apreciado"22. De lo planteado por Carter, autor también de An Introduction to Urban Historical Geography, lo más importante es destacar la influencia ideológica en el plano, poco abordada y apenas tratada en el pensamiento geográfico europeo, cuando desde Europa y más concretamente desde España, desde su cultura, se genera un modelo de ciudad hispanoamericana, ortogonal que obedece a una ideología de raíz cristiana referente a la Jerusalén celestial. (Bielza, "De la ciudad ortogonal..., 2002).

La renovación paisajística de la geografía urbana se produjo desde la influencia que a partir de los sesenta tuvieron las investigaciones de otros expertos de la ciudad sobre la imagen urbana: arquitectos como Kevin Lynch(1960), sociólogos como Ledrut (1973), etc, impulsadas desde la década siguiente por las preocupaciones medioambientales. Geógrafos de formación clásica se hicieron eco de ello, así, la francesa Sylvie Rimbert, en 1973, escribía: "como las cosas, las palabras se usan y se renuevan: la del paisaje acaba de atravesar su periodo demodée que olía a pintor de domingo, para recuperar, a través de las amenazas de las contaminaciones y de los peligros de toda clase una actualidad cierta. En efecto, los cuidados de protección de la naturaleza, de ecología, de urbanismo, de ordenación, que se practican hoy en día, desembocan en paisajes litorales, rurales y urbanos cuya complejidad ha interesado siempre a los geógrafos" (Rimbert, 1973; Bielza, 1975).

Entre los geógrafos anglosajones tuvo un más inmediato y mayor desarrollo una geografía urbana behaviorista, preocupada por el estudio perceptivo del paisaje urbano por parte de los ciudadanos, mediante un mapa mental de estructuración simbólica (semiótica urbana) que condiciona su comportamiento y decisiones. Dicho estudio debe arrojar luz a los planificadores para saber directamente, a través de los propios habitantes, o bien a partir de testimonios aportados por viajeros o escritores, lo que el ciudadano espera de la ciudad y la posibilidad de la mejora de la imagen urbana. Ya fuera del ámbito anglosajón los estudios comportamentales sobre la forma urbana se difunden de tal manera que el profesor de geografía de Besançon A. S. Bailly escribía en 1977 una buena monografía sobre La perception de l'espace urbain, que a los dos años se publicaba en español ${ }^{23} \mathrm{y}$ donde se ocupaba de la percepción del paisaje, el sentido del lugar y la semiótica urbana.

\footnotetext{
${ }^{22}$ En este contexto -señala Carter- quizá uno de los más fascinantes ejemplos de cambio en el plano por cambio cultural está en la cuadratura de Circleville, una ciudad situada en el Estado de Ohio, en los Estados Unidos, que en su origen indio era de forma circular y de trazado radio-concéntrico, obedeciendo -según Reps- a un significado mágico-religioso y que en el siglo XIX los colonizadores europeos fueron cuadriculando.

${ }^{23}$ Bailly, A. S.: La percepción del espacio urbano IEAL, Madrid, 1979. De esta obra son significativos el capítulo I sobre La percepción de los paisajes urbanos, el III sobre El sentido del lugar, el V de La imagen al símbolo y el VI, Técnicas de medición de la percepción del entorno urbano.
} 
La recuperación del lugar urbano, la preocupación por el paisaje existencial, la rehabilitación de los centros históricos son inquietudes que entretanto habían aflorado en la Nueva geografía humanista de Yi-Fu Tuan (1974), Anne Buttimer, Ley, Samuels, y otros (Ley y Samuels, 1978), que se abre camino en los setenta, recomendando la vuelta al historicismo y a Vidal de Blache, aunque desde métodos de investigación en que cuenta más el estudio del ciudadano en relación con su entorno. El chino-norteamericano Yi-Fu tuan, en Topofilia, un estudio de las percepciones, actitudes y valores sobre el entorno abordó el paisaje urbano partiendo de su simbolismo (la ciudad antigua símbolo del cosmos, la metrópoli actual simbolizada por una imagen: la torre Eiffel de París o el skyline de Nueva York) que aunque refleje unos ideales, para la mayoría de las personas, constituye un inmutable, como la naturaleza ${ }^{24}$. Las actitudes hacia los tres tipos de entorno (naturaleza, campo y ciudad) han sido ambivalentes desde el principio. La ciudad preindustrial simbolizaba orden, libertad y gloria, pero también mundanidad, corrupción y opresión. En el occidente, el romanticismo de la naturaleza del XVIII seguido por la revolución industrial; fueron en detrimento de la ciudad. El centro de la gran ciudad se convirtió a partir del XIX en el caos de los parias, mientras que el suburbio, a partir de principios del XX, adquiría atracción y prestigio. La idea de la ciudad jardín o de new town es un intento de combinar las virtudes del modo de vida suburbano con la idea de centro. Los seres humanos han buscado el entorno ideal: el jardín o el cosmos. De este modo, nos movemos de un mundo al otro: de la casa a la plaza pública, del suburbio a la ciudad, buscando un punto de equilibrio que no es de este mundo. A partir de un artículo de David Ley, publicado en Antipode (1976), donde cita la vieja controversia de San Agustín entre la ciudad de Dios y la ciudad de los hombres, Jean-Bernard Racine elabora un libro, La ville entre Dieu et les hommes (1993) que presenta como una reflexión científica sobre la naturaleza, la idea y los modelos de ciudad, tanto en sus símbolos como en sus usos... un análisis de la relación que, en todo tiempo, la ciudad ha tenido con la dimensión de lo sagrado, en su sentido más general, pero también, más particularmente, en el sentido que le ha dado el pensamiento judeocristiano (Racine, 1993).

En los años ochenta, como afirmaba Whitehand, la morfología urbana había retornado a la agenda de la investigación geográfica al resurgir el interés por los lugares, contando con tradiciones como la de Conzen y justificada por el valor económico de las construcciones urbanas de las ciudades industrializadas, por su significado social y como recurso cultural y educativo (Whitehand, 1987). En 1992 el mismo geógrafo anglosajón en unas "Reflexiones sobre la morfología urbana", tras analizar los procesos cíclicos y agentes que cambian el paisaje urbano, destacaba el interés del conocimiento histórico de la forma urbana para resolver la gestión urbanística y la protección del patrimonio cultural (Whitehand, J.W.R. y otros, 1992).

\footnotetext{
${ }^{24}$ La gente siente que sólo controla una pequeña parte de la ciudad. En toda gran metrópolis los estilos de vida varían enormemente. Las personas de la misma ciudad o barrio, perciben mundos diferentes.
} 
En la sociedad post o servoindustrial, que se afirma en Europa en los noventa, la calidad del paisaje urbano y especialmente de los centros, potenciados por la actividad del sector cuaternario, cobra una nueva valoración de cara a la competitividad entre las ciudades, a la hora de atraer la ubicación de las sedes de las multinacionales y de los negocios. Los documentos de la Unión Europea como el de la ETE de Potsdam, 1999, y la Convención Europea del Paisaje, celebrada en Florencia en el 2000 así lo recogen ${ }^{25}$. El paisaje como punto de partida de la morfología urbana aparece en recientes monografías geográficas, como la de H. Capel sobre la Morfología urbana I. Sociedad, cultura y paisaje, donde considera este como un reflejo de la cultura, de la economía y de las funciones urbanas (Capel, 2002, p. 67), para afirmar, más adelante, que la tipología de ciudades según rasgos culturales (china, europea, islámica, iberoamericana) tiene que ver con las formas religiosas que diferencian la configuración de los espacios sagrados o las formas de utilización de la calle y de los espacios públicos en general, con importantes matices en la diferenciación de lo público y de lo privado (Capel, 2002, p. 68).

\section{El nuevo interés de los geógrafos por los centros históricos}

En la era postindustrial los cascos históricos preocupan a todos los científicos de la ciudad, incluidos los geógrafos. La recuperación de los centros históricos es una necesidad para destacar las señas de identidad de las ciudades postindustriales, acrecidas en torno a núcleos preindustriales, puesto que los ensanches de la era industrial y, sobre todo los periurbanos y suburbanos de morfologías estandarizadas son comunes a todas, mientras que lo que define, lo que da la marca a cada ciudad es su centro histórico, con su plano y monumentos. La restauración de dichos centros se ha convertido en una demanda económica de la ciudad postindustrial para competir en el marco de la globalización, atrayendo hacia ellos inversiones de servicios financieros, servicios a las empresas, profesionales, turismo cultural. También es una demanda social de los propios ciudadanos que ven en el casco histórico recuperado una mejora de la calidad de vida para el negocio diurno y el ocio nocturno. La restauración de monumentos, la construcción de vivienda social, la mejora viaria, la peatonalización, la recuperación del pequeño comercio, el saneamiento de los servicios, exigen conocer en los planes especiales de reforma interior, además de las técnicas rehabilitadoras la ideología de los fundadores de la ciudad. Está en juego la sostenibilidad del desarrollo urbano, apoyado en el mantenimiento o la recuperación del patrimonio desde la diversificación de funciones. Es la conclusión a la que llegaba el

\footnotetext{
${ }^{25}$ Comite de desarrollo territorial, U. Europea(1.999): Estrategia Territorial Europea. Hacia un desarrollo equilibrado y sostenible del territorio de la Unión Europea. Luxemburgo, 89 pp. ZOIDO, F. y otros (2002): Paisaje y Ordenación del Territorio. Junta de Andalucía, Sevilla.
} 
geógrafo M. Ferrer en el I Congreso de Centros Históricos de España ${ }^{26}$ (Ferrer, 20001, p. 34). Como afirma el historiador del urbanismo Cesare de Seta la política de conservación del espacio histórico, consolidado por los siglos se funda en una razón ideal con fundamentos económicos y que ha pasado en muchos paises de ser una elección elitista a una opción de masas" lugar", impulsada por la geografía humanística desde finales de los setenta en los países anglosajones y más tarde en el nuestro, supone recuperar la inducción, el historicismo, pero desde una mayor preocupación filosófica y sociológica que la de la geografía vidaliana, como ha señalado Bosque (Bosque, 1986). Así resurge el interés por la geografía histórica y la creciente preocupación teórica y práctica por los centros históricos y la rehabilitación urbana (Norton, W: 1984).

La ciudad reconcebida como lugar, después del furor espacial, recupera los valores culturales, forjados en la historia, desde concepciones ideológicas. La era postindustrial o servo-industrial ha dado un nuevo valor a los cascos históricos como caracterizadores de una ciudad estandarizada y anodina en su periferia industrial. Los centros históricos, debidamente conservados y/o rehabilitados, son factores importantes en la competitividad entre ciudades, dentro de la globalidad. Frente a las ideas de MC Luhan, del "global village", de la aldea global, de que toda la cultura se engloba en un sistema de comunicación que supera las especificidades locales, en la sociedad informacional cada vez es más importante el papel de la cultura local ${ }^{28}$. Castells, ha insistido en que "lo global no disuelve lo local, al contrario, crea la posibilidad de un papel mucho más activo, mucho más decisivo de lo local (Castells y Borja, 1997), ciudad virtual y ciudad real no son excluyentes.

La calidad ambiental del patrimonio cultural-natural, predicada en la Carta de Megárides, impulsada por Beguinot en 1994 y suscrita, entre otros profesionales, por muchos geógrafos, se configura como fundamento de la ciudad sostenible, al mismo tiempo que "cableada". La defensa del patrimonio cultural urbano aparece también recogida en los documentos europeos en materia de ordenación territorial, comenzando por la Carta Europea de 1982-83 (Consejo de Europa, 1983). La "Estrategia Territorial Europea: hacia un desarrollo equilibrado y sostenible de la U.E.", promulgada por la Unión Europea en Postdam (U. Europea1.999) se propuso como uno de

\footnotetext{
26 "En definitiva, parece conveniente devolver al casco la diversificación funcional que es propia de su memoria histórica, integrando las funciones en el presente y el futuro. Esta redefinición de acciones del concepto de rehabilitación reconvirtiéndolo a la pluralidad de acciones, morfológica, social, económica y ambiental, no invalida, sino que enriquece y complementa a la Rehabilitación" (Asoc. para la Recuperación de los Centros Históricos de España).

27 "Por otra parte, ante el fracaso de la ciudad contemporánea -la construida en el último medio sigloresalta la evidencia de que la vieja ciudad (no necesariamente antigua)) normalmente tiene unos valores formales y humanos mucho más convincentes".

${ }^{28}$ Ya Federico Mayor Zaragoza desde la atalaya de la UNESCO se pronunció repetidamente en el sentido de que la globalización económica no debía anular las culturas regionales y locales.
} 
los cinco retos para desarrollar un sistema policéntrico y equilibrado de ciudades la mejora de la calidad urbana, mediante una gestión inteligente y armonizadora de los recursos del ecosistema urbano y contando con la protección y recuperación del patrimonio natural y cultural.

Los centros históricos de la ciudad europea, que en la sociedad informacional hay que proteger y recuperar, se conformaron en buena medida durante la Edad Media, bajo una civilización cristiana ${ }^{29}$. También la ciudad hispanoamericana de la Edad Moderna conoce el mismo origen y su planificación y morfología tiene más que ver con lo experimentado en la península Ibérica en el medioevo que con las doctrinas renacentistas. (Betran, 1992). A comienzos de los noventa hay, al menos, dos obras en España que rompen con la anterior tendencia a marginar las influencias ideológicas en la morfología urbana de los cascos históricos. Una es de un geógrafo, Santiago Quesada, que bajo la dirección de H. Capel, en su tesis doctoral estudia "La idea de ciudad en la cultura hispana de la Edad Moderna". Aunque no trata directamente de la morfología urbana si aborda: la imagen de la ciudad que se desprende de la narración y el valor simbólico de los mitos ${ }^{30} . .$. , una ciudad apostólica basada en las ideas de la Ciudad de Dios" (Quesada, 1992) El otro trabajo, obra del arquitecto Ramón Betrán, se refiere directamente a "La forma de la ciudad. Las ciudades de Aragón en la Edad Media" (Betran, 1992), profundizando sobre la antinomia esbozada por J. M ${ }^{a}$ Lacarra, cuarenta años antes, entre la ciudad islámica y la ciudad cristiana, a partir de los conceptos de orden de ambas religiones y su aplicación a la ciudad ideal, desde la que se desciende a la dispar morfología urbana de ambas. Posteriormente, el que esto suscribe tuvo ocasión de encargar, y prologar otro libro a R. Betrán sobre "El Camino de Santiago y la ciudad ordenada en Aragón”. En dicho prólogo se abría el camino de futuras investigaciones para conectar la morfología urbana, nacida en el siglo XI de la mano del rey Sancho Ramírez en Jaca y Estella, con el urbanismo posterior de la ciudad europea y de la ciudad hispanoamericana, desde la utopía cristiana $^{31}$. En el libro-homenaje al Profesor Ferrer recuperamos la inducción y el historicismo, desde un reflexión filosófica, sociológica y universalizadora para intentar justificar determinadas formas urbanas de los cascos históricos desde concepciones ideológicas cristianas, vertidas, muchas veces, al ordenamiento jurídico para dar satisfacción a unas funciones urbanas, relacionadas a su vez con la situación y que se acoplarán a distintos tipos de emplazamiento (Bielza, 2002). La concepción ideológica

\footnotetext{
${ }^{29}$ Al fin y al cabo, la palabra civilización tiene la misma raíz, "civis", que la de ciudad, "civitas".

30 "Que incorpora, la defensa de ideas filosófico-utópicas sobre la Ciudad Ideal, partiendo de la aplicación a estas obras de la idea de República en Platón y Aristóteles, la difusión de los principios ético-morales de una ciudad apostólica.

${ }^{31}$ Betrán, R.: El Camino de Santiago y la ciudad ordenada en Aragón, 1999. Prólogo de V. Bielza, donde tuve ocasión de adelantar la posible conexión de las tesis de este arquitecto aragonés, que desarrollaba a su vez las del medievalista Lacarra, con las expuestas por el geógrafo francés Bernard y las mías propias, planteadas en Estella, 1968 y después en "La ciudad regular aragonesa y su influencia en el urbanismo regular posterior", 2000, pp. 25-43.
} 
cristiano-medieval aplicada a la forma urbana en las ciudades españolas y americanas gira en torno a la Jerusalén Celestial que se apoya, a su vez, en la Jerusalén prometida al judaísmo por el profeta Ezequiel tal y como han demostrado los geógrafos humanistas Yi-Fu Tuan y Racine.

\section{La forma urbana de los geógrafos planificadores}

La intervención de los geógrafos tanto en la planificación territorial como en la urbana ha servido para enriquecer el pensamiento geográfico en relación con la morfología urbana. Ello ha sido más evidente en el ámbito anglosajón, desde la ya citada obra de Taylor en la primera mitad del siglo XX hasta la de Peter Hall a finales de dicha centuria, con resonancia tanto en la geografía como en la planificación mundial (Hall, P, 1996) En Ciudades del mañana Hall diferencia los modelos que más han impactado en las planificaciones del siglo XX para ordenar la ciudad industrial: la ciudad-jardín, la ciudad-región, la ciudad de las torres o bloques (Le Corbusier) y la ciudad dispersa de viviendas unifamiliares (Wright). Además hay que seguir contemplando el monumentalismo de exaltación del poder, que ya existía en la preindustrial.

A partir de la crisis del 73 surgirá la necesidad de ordenar la ciudad postindustrial. La regeneración de frentes de agua en el Old Port bostoniano en 1976, mediante la sustitución de los decadentes usos del suelo portuario-industriales por los residenciales, comerciales y lúdicos, contando con la cooperación del Estado, la municipalidad y los promotores privados, inauguró una nueva fórmula urbanística, utilizada en el viejo continente para salir de la crisis de los años setenta. Concretamente en los docklands (muelles) de Londres convergieron en los ochenta las experiencias norteamericanas con las ideas del geógrafo Peter Hall de crear allí zonas de negocios, utilizando la figura de puerto franco que tanto éxito tenía en Hong Kong. Desde la batalla de los muelles londinenses hemos asistido a reconversiones de muchas zonas portuarias europeas y luego de algunas sudaméricanas (Puerto Madero). La experiencia de los water front ha sido recogida por las grandes Exposiciones postmodernas en las que se han aprobado diez principios para el desarrollo sostenible del waterfront , base del modelo de la ciudad del futuro sobre suelo nuevo, que se está haciendo realidad en Waterfront City, Dubai, alargando 70 kilómetros la línea de la costa (Bielza, 2011).

\section{Bibliografía citada}

Bastié et Dezert: L'espace urbain. Masson. Beaujeu-Garnier et Chabot: Traité de Paris, 1980. Geographie urbaine. A. Colin, Paris, 1963.

Bailly, A.S.: La percepción del espacio urbano Berdoulay, V.: Aux débuts de l'urbanisme IEAL, Madrid, 1979. français. L'Harmattan, Paris, 2001. 
Berdoulay, V. et Soubeyran, O.: L'Ecologie urbaine et l'urbanisme. Aux fondements des enjeux actuels. La Découverte, Paris, 2002

Betran, R.: La forma de la ciudad. Las ciuda des de Aragón en la Edad Media. Col Arquitectos, Zaragoza, 1992, 510 pp.

Betrán, R.: El Camino de Santiago y la ciudad ordenada en Aragón, Gob. Aragón, 1999 Zaragoza. Prólogo de V. Bielza.

Bielza, V. Estella, estudio geográfico de una pequeña ciudad navarra. P de Viana Pamplona, 1968.

Bielza, V.: "Una visión geográfica acerca de la degradación del paisaje y los problemas de medio ambiente", Cuad. de Investigación, tomo I, 2, Logroño, 1975.

Bielza de Ory, V.:" La ciudad regular aragonesa y su influencia en el urbanismo regular posterior". Aragón en la Edad Media, XVI, Univ. Zaragoza, Zaragoza, 2000.

Bielza, V.: "De la ciudad ortogonal a a la cuadricular hispanoamericana como proceso de innovación-difusión, condicionado por la utopía" Scripta Nova Rev. Geocrítica. Univ. de Barcelona. Vol. VI, no 106, 2002.

Bielza de Ory, V.:"Notas sobre la morfología urbana de los cascos históricos europeos y la utopía cristiana". Homenaje a Manuel Ferrer Regales, EUNSA, Pamplona, 2002, $1.191 \mathrm{pp}$.

Bielza de Ory, V.:Los nuevos paisajes urbanos de waterfront $y$ las grandes exposiciones postmodernas: un modelo de la ciudad del futuro. Comunicación a la Conferencia Regional de la UGI, Actas. Santiago de Chile, 2011

Blanchard, R,: Grenoble, étude de géographie urbaine. Paris., 1912. A. Colin.

Bosque Maurel, J.: "El espacio urbano. Evolución y cambio en geografía urbana" pp 259-283 del libro de A. García Ballesteros: Teoría y práctica de la Geografia Alambra Univ. Madrid, 1986.

Bosque Maurel, J. "Geografía, historia y Geografía histórica", Estudios Geográficos XLIV, 1983, páginas 317-338.

Bunge; W: Theoretical geography, 1962.

Capel,H.: La morfología de las ciudades .Ed. Serbal, Barelona, 2002.

Carter, H. The study of Urban Geography. Col. Univ. de Gales. Aberystwyth Arnold. Londres 1972. Madrid, 1974.

Carter, H.: An Introduction to Urban Historical Geography, Londres, E. Arnold, 222, pp.

Castells, M. y Borja, J.: "Local y global". Taurus, 1997.

Consejo de Europa (1983): Carta Europea de Ordenación del Territorio. EstrasburgoTorremolinos.

Comite de desarrollo territorial, U. EUROPEA(1.999): Estrategia Territorial Europea. Luxemburgo, 89 pp.

Derruau, M.: Précis de Geographie humaine. A. Colin, Paris, 1961.

Dickinson, R.E.: The West European city . A Geographical Interpretation. Routledge. Londres, 1951.

Estébanez, J.: "Las ciudades" en Geografia General II . Ed. Taurus. Madrid, 1993, dir. V. Bielza.

George P.: Précis de geographie urbaine (PUF, Paris, 1960).

Ferrer Regales, M.: I Congreso de Centros Históricos de España. Centro Cultural Villa de Madrid. 20001, pp. 21-49.

Hagget, P.: Locational Analysis in Human Geography. Ed. E.Arnold.Londres, 1965.

Haggett, P.: Geography:A Modern Synthesis. Ed. Harper, New York, 1983. 
Hall, Peter: Ciudades del mañana. Ed. Serbal, Barcelona, 1996.

Hassinger, H.: Kunsthistorischer Plan des I Berzziks der Städt Wien, Viena, 1912.

Jonson, J.H.: Urban Geography: an introductory Análisis. Pergamon. Oxford, 1974. Oikos-tau, Barcelona, 1974.

Lavedán, P: Historie de l’urbanisme, 4 vols., Paris, 1916-52. Geographie des villes: Paris, 1936.

Ledrut, R.: Les images de la ville. Anthropos. Paris, 1973.

Ley, D. y Samuels, M.S. Humanistic Geography. Prospects and Human geography Ed. Croom Helm,Londres, 1978.

Lynch, K: The image of the city. Cambridge. Hass, 1960.

Krebs, N: Geografía Humana. Ed. Labor, Barcelona, 1931. Trad. G. Reparaz.

Norton, W: Historical Analysis in Ceography, Londres, Longman, 1984, 231 pp.

Quesada, S.:"La idea de ciudad en la cultura bispana de la Edad Moderna".Univ. Barcelona. 1992 (Geocrítica).

Racine J-B.: La ville entre Dieu et les hommes. Ed. Anthopos. Economica. Paris, Ginebra, 1993.

Seta, C. De: La città europea dal XV al XX secolo. Origini, sviluppo e crisi della civiltà urbana in età moderna e contemporanea. RCS. Libri\&Grandi Opera. S.p.A., Milan, 1996.
Ratzel, Fr: Anthropogeographie. 2 partes, bibliothek geog. Stuttgart-1882-18 Barcelona.

Schlüter,O.: "Ueber den Grundriss der Städte". Zeitschrift der Gesellschaft für Erkunde zur Berlin XXIV, 1899.

Reps, J. W. The making of urban America, Princenton, 1965.

Rimbert, S.: "Approches des paysages". Rev L'espace geographique, t. II, no 3, 1973 pp. 233-241.

Taylor, Griffith: Urban Geography. Toronto, 1946. Trad. Esp. Geografía urbana. Un estudio del emplazamiento, evolución, forma y clasificación de pueblos, villas y ciudades, $495 \mathrm{pp}$.

Tricart, J. L' habitat urbain. Cours. de Geogr. humaine. Strasburg, 1954

Whitehand, J.W.R.: The Changing Face of Cities: A Study of Development Cycles and Urban Form. Inst. of British Geographers Special Publications $\mathrm{n}^{\circ}$ 21, Ed. Basil Blackwell, Oxford, 1987.

Whitehand, J.W.R. y otros: Morfología y paisajes urbanos. La perspectiva geográfica británica. Ed. Univ. Lleida, 1992.

Yi-Fu tuan, Topophilia. A study of environmental perception, attitudes and values, Prentice-Hall Inc., New Jersey 1974.

Zoido, F.:Prólogo . Paisaje y Ordenación del Territorio. Cons. de O. Públicas. Junta de Andalucía, Sevilla, 2002 